Running Head: PERCEIVED SEVERITY OF THE COVID-19 AND MENTAL HEALTH

\title{
Self-control moderates the association between perceived severity of the coronavirus disease 2019 (COVID-19) and mental health problems among the Chinese public
}

\author{
Jian-Bin Li \\ Department of Early Childhood Education, Center for Child and Family Science, \\ The Education University of Hong Kong, Hong Kong, S.A.R. China
}

An Yang

Department of Applied Psychology, Guangdong University of Foreign Studies, Guangzhou, P. R. China

Kai Dou*

Department of Psychology and Research Center of Adolescent Psychology and Behavior, School of Education, Guangzhou University, Guangzhou, P. R. China

Rebecca Y. M. Cheung

Department of Early Childhood Education, Center for Child and Family Science, Center for Psychological Health, The Education University of Hong Kong, Hong Kong, S.A.R. China

\footnotetext{
* Corresponding author:

Kai Dou, Ph.D.

Associate Professor

Guangzhou University

230, Waihuan Road West,

Panyu District, Guangzhou, P. R. China

E-mail: psydk@gzhu.edu.cn
} 


\section{Acknowledgements of financial support}

Jian-Bin Li was supported by the FEHD's Internationalization \& Exchange Research Scheme of the Education University of Hong Kong. Kai Dou was support by the MOE (Ministry of Education in China) Project of Humanities and Social Sciences (17YJCZH040). 


\begin{abstract}
The coronavirus disease 2019 (COVID-19) has caused thousands of death in China. Prior research suggests that individuals' perceived severity of the COVID-19 is related to a range of negative emotional and behavioural reactions among the Chinese public. However, scant research has examined the underlying mechanisms. Drawing upon the risk-resilience model, this study proposes that self-control, as a resilient factor, would potentially moderate the association between perceived severity of the COVID-19 and mental health problems. Data from a national survey was used to examine this possibility. Participants were 4,607 citizens from 31 regions in China $\left(M_{\text {age }}=23.71\right.$ years, $72.5 \%$ female $)$ who completed a national survey at the beginning of February 2020. Results of hierarchical regression showed that after controlling for demographic variables, perceived severity of the COVID-19 and self-control were positively and negatively related to mental health problems, respectively. More importantly, self-control moderated the "perceived severity of the COVID-19 - mental health problems" association, with the said link attenuating as the levels of self-control increased. These findings suggest that compared to those with high self-control, individuals with low self-control are more vulnerable and are more in need of psychological aids to maintain mental health in the encounter of the COVID-19 outbreak.
\end{abstract}

Key words: risk factor; resilience; cognitive appraisal; self-control; COVID-19; public health concerns. 


\section{Self-control moderates the association between perceived severity of the coronavirus disease 2019 (COVID-19) and mental health problems among the Chinese public}

The outbreak of the coronavirus disease 2019 (COVID-19) has not only led to thousands of death in China, but it also caused tremendous psychological stress to the Chinese public. In order to medically and mentally combat the COVID-19, professional input from various disciplines is needed, including the perspectives from psychological science. A recent study conducted among 4,607 Chinese reveals that individuals' cognitive appraisals, perceived severity of the COVID-19 in particular, are related to a number of undesirable emotional (e.g., increase in negative emotion) and behavioural (e.g., increase in sleep problems) reactions (Authors, 2020) ${ }^{1}$. However, little is known about what factors can buffer the negative influence of perceived severity on individuals' mental health. Studying this issue is important and necessary, as the findings will shed light on at specific factors for intervention programs to target. Drawing upon the risk-resilience model (Masten, 2001), we proposes that self-control ------ one's ability to override or change inner responses and to interrupt undesired behavioural tendencies (Tangney, Baumeister, \& Boone, 2004) ------ could be a candidate that buffers the negative influence of perceived severity of the COVID-19 and mental health problems among the Chinese public. The aim of this study is to examine this idea.

The risk-resilience model proposes that risk and adversity increase the propensity of undesirable outcomes; individuals who have sufficient assets to offset the negative influence of the risk could overturn undesirable outcomes, thus showing resilience (Masten, 2001). In this model, risk can be defined in diverse ways, including both intrinsic and extrinsic factors such as

\footnotetext{
${ }^{1}$ Names are omitted for blind review purpose but will be released once the paper is accepted.
} 
adverse living condition, the negative life events that have occurred in recent months, massive community trauma, and cumulative risk calculations that combine different kinds of risk factors. The main effect model suggests that risk factors are associated with undesirable outcomes such as maladjustment. Nevertheless, the compensatory effect (or moderation effect) assumes that enough positive internal and/or external assets could mitigate the burden of an individuals' life due to risk influences, and thus these individuals would have better outcomes than those who have the same levels of risk but do not possess enough assets.

A recent study reveals that individuals' perceived severity of the COVID-19 outbreak is related to more undesirable emotional and behavioural outcomes in the Chinese public (Authors, 2020), suggesting that perceived severity of the COVID-19 may impose a negative main effect on mental health outcomes. This is in line with past studies which found that individuals' negative appraisals about the incident (e.g., perceived risk, perceived threat, etc.) were related to more mental health problems during the outbreak of the severe acute respiratory syndrome (SARS) and the Ebola (Cheng et al., 2006; Dorfan \& Woody, 2011; Vartti et al., 2009; Yang \& Chu, 2018). These literatures mainly adopt the main effect model to examine the risk and protective factors of outcomes during the encounter of emergent public health concerns. Beyond this, the risk-resilience model suggests the necessity and importance to take into account how assets may buffer the negative main effect on health outcomes. This motivated us to examine the potential moderation effect of self-control.

As an asset, self-control ability may serve to regulate the undesirable mental health consequences brought by the COVID-19. Previous research suggested that individuals with high self-control have better inhibition and initiatory ability (de Ridder, de Boer, Lugtig, Bakker, \& van Hooft, 2011), use more positive coping strategies and fewer negative coping strategies ( $\mathrm{Li}$, 
Delvecchio, Lis, Nie, \& Di Riso, 2016), and persist more in important life domains (Duckworth \& Seligman, 2005; Moffitt et al., 2011). In addition, self-control supports the retrieval of restrain standards and deliberative evaluations (Hofmann, Friese, \& Strack, 2009). Therefore, good selfcontrol is robustly related to a wide range of life outcomes, including better mental health (for a review, see de Ridder, Lensvelt-Mulders, Finkenauer, Stok, \& Baumeister, 2012). Besides, several aspects of good self-control are particularly relevant to the moderation in the association between risk factors and health outcomes. For instance, planning and forethought could help individuals anticipate and prepare for difficult situations; emotional self-regulation could provide better emotional control in problem situations; the ability to restrain and initiate action is important to deal with the difficulties in problem situations (Wills, Ainette, Stoolmiller, Gibbons, \& Shinar, 2008). Prior studies have found that good self-control buffers the influence of negative environment (e.g., peer deviance, negative life events) on undesirable outcomes such as substance use (Dishion \& Connell, 2006; Wills et al., 2008). This suggests that self-control is not only directly related to mental health but may also moderate the negative effect of risk factors, such as perceived severity.

\section{The current study}

According to the literature reviewed above and the logic of the risk-resilience model, we hypothesized that perceived severity of the COVID-19 and self-control would be related to mental health problems in positive and negative directions, respectively. Moreover, we assumed that the negative association between perceived severity of the COVID-19 and mental health problems would be less pronounced among those with high self-control compared to their lowself-control counterparts. To examine these hypotheses, we used data from a recent national survey (Author, 2020) which examines the Chinese public's emotional and behavioural 
outcomes and its antecedents in the encounter of the COVID-19 outbreak. Given that the individual data were nested in regions in this dataset, we employed a hierarchical regression analysis to control for the potential influence of the cluster, with a number of demographic variables included in the model as covariates to rule out their influence on the outcome.

\section{Method}

\section{Participants and procedure}

Between February 2 and 9, 2020, 4826 Chinese individuals visited our online survey website. A total of 219 participants were excluded because they met one of the following criteria: (1) they did not show interest to participate in the study after reading the information sheet; (2) they were not old enough to provide consent form (i.e., $\leqq 16$ years); or (3) they showed an obvious responding pattern across multiple consecutive items (e.g., select " 3 " for all items). Thus, 4607 participants comprised the final sample, 1265 males (27.5\%) and 3342 females (72.5\%) with age ranging from 17 to 90 years $\left(\mathrm{M}_{\mathrm{age}}=23.71\right.$ years, $\left.\mathrm{SD}=7.29\right)$. These participants came from 31 regions in China, with the sample size ranging from $16(0.3 \%$ of the total sample, Ningxia Hui Autonomous Region) to 1386 (30.1\% of the total sample, Guangdong Province). Most participants (73.2\%) held a bachelor degree or above. Regarding physical and mental health condition, $77.3 \%$ of participants reported that their current physical health was good or very good; $94.6 \%$ and $99.2 \%$ of participants reported that they did not have any history of chronic physical diseases or history of psychiatric / psychological disorders, respectively. In addition, $97.7 \%$ of participants reported they had not been diagnosed with the COVID-19; $2.3 \%$ of participants reported they were suspected / diagnosed with the COVID-19 or had relatives / friends who were suspected / diagnosed cases. 
The ethical committee of the Guangzhou University reviewed and approved this study before data collection. This study was part of the large survey study which aimed to examine Chinese public's emotional and behavioural outcomes and its risk and protective factors towards the COVID-19 outbreak (Authors, 2020). Over 200 volunteers who majored in psychology helped distribute the survey link on various internet platforms, including WeChat, Weibo, QQ, Facebook, etc. after receiving a three-hour training. Informed consent was sought prior to participation. Voluntary participation was emphasized and no incentive reward was given. We also stressed anonymity and did not collect any identifiable personal particulars.

\section{Measures}

Mental health problems. The Chinese version of the 12-item General Health Questionnaire (GHQ-12, Zhang \& Wang, 2014) was used to measure participants' overall mental health problems over the past ten days. The scale contains 12 items either in negative or positive wordings. Participants were asked to indicate their situation on these items over the past 10 days compared to their general situation. All items were rated on a 4-point scale. The first two options of each question indicate that participants feel better than, or as much as, usual. Thus, these two options were coded 0 . The third and the fourth option indicate participants feel/experience a little and much poorer than usual, respectively, and thus they were coded 1 , and 2 , respectively. Therefore, a higher total score reflects more mental health problems. Sample items are "feeling unhappy and depressed" (negative wording) and "been able to face up to your problems" (positive wording). The Cronbach's $\alpha$ was 0.86 in this study.

Perceived severity. Participants' perceived severity about the COVID-19 was measured with 5 items. Participants indicated their evaluation of how severe they think of the COVID-19 is in various aspects on a five-point scale (from $1=$ not severe at all to $5=$ very much severe). A 
higher score indicates participants perceived the COVID-19 to be more severe. Sample items are "how severe you think of the infectious rate of the COVID-19 is?" and "how severe you think of the morbidity of the COVID-19 is?" The Cronbach's $\alpha$ was 0.84 in this study.

Self-control. The Chinese version of the Brief Self-Control Scale (BSCS) was used to measure participants' self-control ability (Li, Vazsonyi, \& Dou, 2018). The scale consists of 13 items rated on a five-point scale (from 1=not like me at all to 5=like me very much). A higher mean score indicates better self-control ability. Sample items are "I am good at resisting temptation" and "I have a hard time breaking bad habits". The Cronbach's $\alpha$ was 0.84 in this study.

\section{Data analysis}

We analysed the data in SPSS and Mplus 7.0. First, we carried out descriptive statistics to capture the centrality of the variables. Second, we performed correlation analysis to examine the association between perceived severity, self-control and mental health problems. Last, given that the individual data were nested in different provinces/regions, we carried out hierarchical regression models to examine the moderation effect of self-control in the association between perceived severity and mental health problems, controlling for demographic variables. To this end, we first calculated the intraclass correlation and the variance at the within- and betweenlevel. Then, we centred the covariates, independent variable and the moderator using the "groupmean" function (Enders \& Tofighi, 2007). Subsequently, we calculated the product term between the centred independent variable and the centred moderator as the interaction term. Based on this, we fit a full model, with the independent variable, the moderator, the interaction term, and the covariates as level-1 variables. The intercept of the dependent variable was also estimated at level-2 to control for the variance of the dependent variable accounted for by the 
cluster. Finally, we conducted simple slope tests with 1SD below and above the centred mean of the moderator and examined the differences in the magnitude of the slopes. Since Mplus 7.0 does not accommodate bootstrapping with multi-level models, we used $\mathrm{R}$ to calculate the $95 \%$ bootstrapping $(N=20,000)$ confidence interval of the simple slopes and their differences.

\section{Results}

Mean levels of and the bivariate correlations between mental health problems, perceived severity, and self-control

As shown in Table 1 , participants reported only a few mental health problems $(0.19$ out of 2) over the past 10 days, relatively high levels of perceived severity of the COVID-19 (4.09 out of 5) and medium levels of self-control (3.03 out of 5). The results of correlation analyses found that perceived severity of the COVID-19 and self-control was negatively and positively related to mental health problems, respectively.

\section{[INSERT TABLE 1 ABOUT HERE]}

\section{The moderation of self-control in the association between perceived severity and mental health problems}

We conducted hierarchical regression models to examine the association between perceived severity of the COVID-19 and mental health problems as well as the moderation of self-control. Results of the null model revealed that the intraclass correlation (ICC) of mental health problems was 0.04 . The within-level and the between-level model explained $7.1 \%$ and $0.3 \%$ variance of mental health problems, respectively. 
The results of the full model are summarized in Table 2 . The results showed that after controlling for a number of demographic variables, the main effect of perceived severity on mental health problems was significant $(B=0.07, S E=0.01, p<0.001)$, suggesting that participants who perceived the COVID-19 to be more severe reported more mental health problems. The main effect of self-control was also significant $(B=-0.08, S E=0.01, p<0.001)$, suggesting that participants with high self-control reported fewer mental health problems. More importantly, the interaction effect between perceived severity and self-control was significant as well, $B=0.05$, $S E=0.01, p<0.001$.

\section{[INSERT TABLE 2 ABOUT HERE]}

Given the significance of the interaction term, we further conducted simple slope tests and the results are displayed in Table 3 and Figure 1. The results showed that as individuals' self-control ability increased, the association between perceived severity and mental health problems decreased. Furthermore, we compared the differences in the magnitude of the simple slopes, finding that the slope of perceived severity at the low levels of self-control was significantly larger than the ones at the medium and high levels of self-control and that the slope at the medium levels of self-control was also significantly larger than the one at the high levels of self-control. 


\section{Discussion}

The COVID-19 has been declared as an emergent public health concern (American, 2020). Prior research found that perceived severity of the COVID-19 was associated with more undesirable emotional and behavioural outcomes (Author, 2020). Deepening this issue, the current study sought to examine self-control as a candidate to moderate the association between perceived severity and mental health problems among the Chinese public. The findings fully supported our hypotheses, even after controlling for a range of demographic variables.

This study bears both theoretical and clinical implications. Theoretically, the significant interaction suggests that besides the main effect model, the interaction effect model is also important in studying mental health in the context of emergent public health concerns. This is because the effect of a risk factor on health outcomes could be masked or averaged without examining its interaction with other risks and assets, and thereby the likelihood of detecting such an effect is reduced. Clinically, high levels of self-control buffers the association between perceived severity and mental health problems, suggesting that individuals with low levels of self-control are more vulnerable. This implies that those with low levels of self-control would be more in need of psychological aids, which echoes the call by the Chinese government "to provide mental aid to those who need it". In addition, since self-control is a crucial asset, enhancing individuals' self-control ability may help buffer the influence of negative appraisal and improve the public's mental health.

We must acknowledge that the current study has several limitations. First, the self-report data increases the common method bias and the cross-sectional nature prevents us from inferring causality. Second, samples from different provinces/regions vary greatly, which may affect the estimation of the between-level variance. Third, some populations were over-representative, such 
as females and students. A more sophisticated design and more representative sample would be desirable to achieve even robust findings. Nevertheless, the current findings provide important early evidence about the mental health problems in the Chinese public and how these problems are jointly explained by both risk (i.e., perceived severity) and asset (i.e., self-control) during the COVID-19 outbreak. As the virus currently is spreading worldwide, the current findings could be of potential use for further research around the globe. In conclusion, perceived severity of the COVID-19 relates to more mental health problems and high levels of self-control buffer this association. We believe that these findings bear important implications in understanding and improving the public's mental health during the encounter of emergent public health concerns.

\section{Reference}

Authors [omitted for blind review]. (2020). Chinese public's knowledge, perceived severity, and perceived controllability of the COVID-19 and their associations with emotional and behavioural reactions, social participation, and precautionary behaviour: A national survey. Manuscript under review.

American, S. (2020). WHO declares coronavirus outbreak a global health emergency. Retrieved from https://www.scientificamerican.com/article/who-declares-coronavirus-outbreak-aglobal-health-emergency/

Cheng, S. K. W., Chong, G. H. C., Chang, S. S. Y., Wong, C. W., Wong, C. S. Y., Wong, M. T. P., \& Wong, K. C. (2006). Adjustment to severe acute respiratory syndrome (SARS): Roles of appraisal and post-traumatic growth. Psychology \& Health, 21(3), 301-317. doi:10.1080/14768320500286450

de Ridder, D. T. D., de Boer, B. J., Lugtig, P., Bakker, A. B., \& van Hooft, E. A. J. (2011). Not doing bad things is not equivalent to doing the right thing: Distinguishing between inhibitory and initiatory self-control. Personality and Individual Differences, 50(7), 10061011. doi:https://doi.org/10.1016/j.paid.2011.01.015

de Ridder, D. T. D., Lensvelt-Mulders, G., Finkenauer, C., Stok, F. M., \& Baumeister, R. F. (2012). Taking stock of self-control: a meta-analysis of how trait self-control relates to a 
wide range of behaviors. Personality and Social Psychology Review, 16(1), 76-99. doi:10.1177/1088868311418749

Dishion, T. J., \& Connell, A. (2006). Adolescents' resilience as a self-regulatory process: promising themes for linking intervention with developmental science. Annals of the New York Academy of Sciences, 1094, 125-138. doi:10.1196/annals.1376.012

Dorfan, N. M., \& Woody, S. R. (2011). Danger appraisals as prospective predictors of disgust and avoidance of contaminants. Journal of Social and Clinical Psychology, 30(2), 105132. doi:10.1521/jscp.2011.30.2.105

Duckworth, A. L., \& Seligman, M. E. P. (2005). Self-discipline outdoes IQ in predicting academic performance of adolescents. Psychol Sci, 16(12), 939-944. doi:10.1111/j.14679280.2005.01641.x

Enders, C. K., \& Tofighi, D. (2007). Centering predictor variables in cross-sectional multilevel models: A new look at an old issue. Psychological Methods, 12(2), 121-138. doi:10.1037/1082-989x.12.2.121

Hofmann, W., Friese, M., \& Strack, F. (2009). Impulse and self-control from a dual-systems perspective. Perspectives on Psychological Science, 4(2), 162-176. doi:10.1111/j.17456924.2009.01116.x

Li, J.-B., Delvecchio, E., Lis, A., Nie, Y.-G., \& Di Riso, D. (2016). Positive coping as mediator between self-control and life satisfaction: Evidence from two Chinese samples. Personality and Individual Differences, 97, 130-133. doi:10.1016/j.paid.2016.03.042

Li, J.-B., Vazsonyi, A. T., \& Dou, K. (2018). Is individualism-collectivism associated with selfcontrol? Evidence from Chinese and U.S. samples. PLoS One, 13(12), e0208541. doi:10.1371/journal.pone.0208541

Masten, A. S. (2001). Ordinary magic. Resilience processes in development. The American psychologist, 56(3), 227-238. doi:10.1037/0003-066x.56.3.227

Moffitt, T. E., Arseneault, L., Belsky, D., Dickson, N., Hancox, R. J., Harrington, H., . . Caspi, A. (2011). A gradient of childhood self-control predicts health, wealth, and public safety. Proceedings of the National Academy of Sciences of the United States of America, 108(7), 2693-2698. doi:10.1073/pnas.1010076108 
Tangney, J. P., Baumeister, R. F., \& Boone, A. L. (2004). High self-control predicts good adjustment, less pathology, better grades, and interpersonal success. Journal of Personality, 72(2), 271-324. doi:10.1111/j.0022-3506.2004.00263.x

Vartti, A. M., Oenema, A., Schreck, M., Uutela, A., de Zwart, O., Brug, J., \& Aro, A. R. (2009). SARS Knowledge, Perceptions, and Behaviors: a Comparison between Finns and the Dutch during the SARS Outbreak in 2003. International Journal of Behavioral Medicine, 16(1), 41-48. doi:10.1007/s12529-008-9004-6

Wills, T. A., Ainette, M. G., Stoolmiller, M., Gibbons, F. X., \& Shinar, O. (2008). Good SelfControl as a Buffering Agent for Adolescent Substance Use: An Investigation in Early Adolescence With Time-Varying Covariates. Psychology of Addictive Behaviors, 22(4), 459-471. doi:10.1037/a0012965

Yang, J. Z., \& Chu, H. R. (2018). Who is afraid of the Ebola outbreak? The influence of discrete emotions on risk perception. Journal of Risk Research, 21(7), 834-853. doi:10.1080/13669877.2016.1247378

Zhang, L., \& Wang, J. (2014). The introduction of the Common Mental Health Screening Scales and the comparison of their reliablity and validity. Chinese Journal of Social Medicine, $31(2), 86-88$. 
Table 1

Descriptive statistics and bivariate correlations of mental health problems, perceived severity, self-control, and demographic variables

\begin{tabular}{|c|c|c|c|c|c|c|c|c|c|c|}
\hline & 1 & 2 & 3 & 4 & 5 & 6 & 7 & 8 & 9 & 10 \\
\hline \multicolumn{11}{|l|}{ 1. Sex } \\
\hline 2. Age & $-0.08^{* * *}$ & & & & & & & & & \\
\hline 3. Phy.history & $0.06^{* * *}$ & $-0.10^{* * *}$ & & & & & & & & \\
\hline 4. Psy.history & -0.01 & 0.01 & $0.08^{* * *}$ & & & & & & & \\
\hline 5. Health con. & $-0.04^{* *}$ & -0.03 & $0.18^{* * * *}$ & $0.08^{* * *}$ & & & & & & \\
\hline 6. Education & -0.00 & $-0.13^{* *}$ & $-0.03^{*}$ & $0.03^{*}$ & 0.02 & & & & & \\
\hline 7. Rel. w. COVID-19 & 0.01 & $0.09^{* *}$ & -0.02 & $-0.03^{*}$ & $-0.03^{*}$ & -0.01 & & & & \\
\hline 8. Mental health problems & $0.07^{* * *}$ & $0.04^{*}$ & $-0.08^{* * *}$ & $-0.09^{* * *}$ & $-0.19^{* * *}$ & 0.02 & $0.03^{*}$ & & & \\
\hline 9. Perceived severity & $0.11^{* * *}$ & $0.09^{* * *}$ & 0.00 & -0.00 & $-0.04^{* *}$ & $-0.03^{*}$ & -0.00 & $0.19^{* * *}$ & & \\
\hline 10. Self-control & -0.01 & $0.21^{* * *}$ & $0.05^{* *}$ & $0.06^{* * *}$ & $0.21^{* * *}$ & $-0.06^{* * *}$ & 0.03 & $-0.21^{* * *}$ & $-0.10^{* * *}$ & \\
\hline Min & 1.00 & 17 & 1.00 & 1.00 & 1.00 & 1.00 & 1.00 & 0.00 & 1.00 & 1.31 \\
\hline $\operatorname{Max}$ & 2.00 & 90 & 2.00 & 2.00 & 5.00 & 6.00 & 2.00 & 2.00 & 5.00 & 5.00 \\
\hline$M$ & 1.73 & 23.71 & 1.95 & 1.99 & 4.06 & 3.68 & 1.02 & 0.19 & 4.09 & 3.03 \\
\hline$S D$ & 0.45 & 7.29 & 0.23 & 0.09 & 0.75 & 0.77 & 0.15 & 0.27 & 0.59 & 0.50 \\
\hline
\end{tabular}

Note.

Sex: 1=male, $2=$ female;

Phy.history $=$ history of chronic physical diseases: $1=y e s, 2=$ no;

Psy.histroy $=$ history of psychiatric / psychological disorder: $1=y e s, 2=n o$;

Health con. $=$ current physical health condition, from $1=$ very poor to $5=$ very good;

Education: $1=$ junior middle school and below, $2=$ high school degree, $3=$ college degree, $4=$ bachelor degree, $5=$ master degree, and $6=$ doctoral degree.

Rel. w. COVID-19 = relationship with the COVID-19: 1=not relevant / infected, $2=$ suspected / diagnosed cases or had relatives / friends who were suspected / diagnosed cases.

${ }^{*} p<0.05 ;{ }^{* *} p<0.01 ;{ }^{* * *} p<0.001$ 
Table 2

Hierarchical regression model of the association between perceived severity and mental health problems and the moderation effect of self-control

\begin{tabular}{llll}
\hline & $B$ & $S E$ & \\
\hline Sex & $B$ & 0.01 & \\
Age & 0.04 & 0.00 & 0.02 \\
Phy.history & 0.00 & 0.048 \\
Psy.history & -0.04 & 0.07 & 0.025 \\
Health con. & -0.17 & 0.01 & 0.01 \\
Education & -0.04 & 0.03 & 0.01 \\
Rel. w. COVID-19 & 0.01 & 0.01 & 0.013 \\
Perceived severity & 0.07 & 0.01 & 0.01 \\
Self-control & -0.08 & $<0.001$ \\
Perceived severity $\times$ self-control & -0.05 & $<0.001$ \\
\hline
\end{tabular}

Note.

Sex: $1=$ male, $2=$ female;

Phy.history $=$ history of chronic physical diseases: $1=y e s, 2=$ no;

Psy.histroy $=$ history of psychiatric / psychological disorder: 1=yes, 2=no;

Health con. $=$ current physical health condition, from $1=$ very poor to $5=$ very good;

Education: $1=$ junior middle school and below, $2=$ high school degree, $3=$ college degree, $4=$ bachelor degree, $5=$ master degree, and $6=$ doctoral degree. Rel. w. COVID-19 = relationship with the COVID-19: 1=not relevant / infected, $2=$ suspected / diagnosed cases or had relatives / friends who were suspected / diagnosed cases. 
Table 3

Summary of simple slopes tests by the levels of self-control and the difference in simple slopes

\begin{tabular}{|c|c|c|c|c|}
\hline & $B$ & $S E$ & $p$ & $95 \%$ bootstrapping $C I(N=20000)^{\mathrm{b}}$ \\
\hline \multicolumn{5}{|l|}{ Simple slopes } \\
\hline Low self-control (-1SD) & 0.10 & 0.009 & $<0.001$ & {$[0.080,0.116]$} \\
\hline Medium self-control (mean) ${ }^{\mathrm{a}}$ & 0.07 & 0.007 & $<0.001$ & {$[0.058,0.086]$} \\
\hline High self-control (1SD) & 0.05 & 0.009 & $<0.001$ & {$[0.028,0.064]$} \\
\hline \multicolumn{5}{|l|}{ Comparison between simple slopes } \\
\hline Difference between low and medium self-control & 0.03 & 0.006 & $<0.001$ & {$[0.015,0.037]$} \\
\hline Difference between low and high self-control & 0.05 & 0.012 & $<0.001$ & {$[0.029,0.075]$} \\
\hline Difference between medium and high self-control & 0.04 & 0.006 & $<0.001$ & {$[0.015,0.037]$} \\
\hline
\end{tabular}

Note.

a: Medium self-control refers to the centred mean (i.e., 0.00).

b: $95 \%$ CIs were calculated with $\mathrm{R}$. 


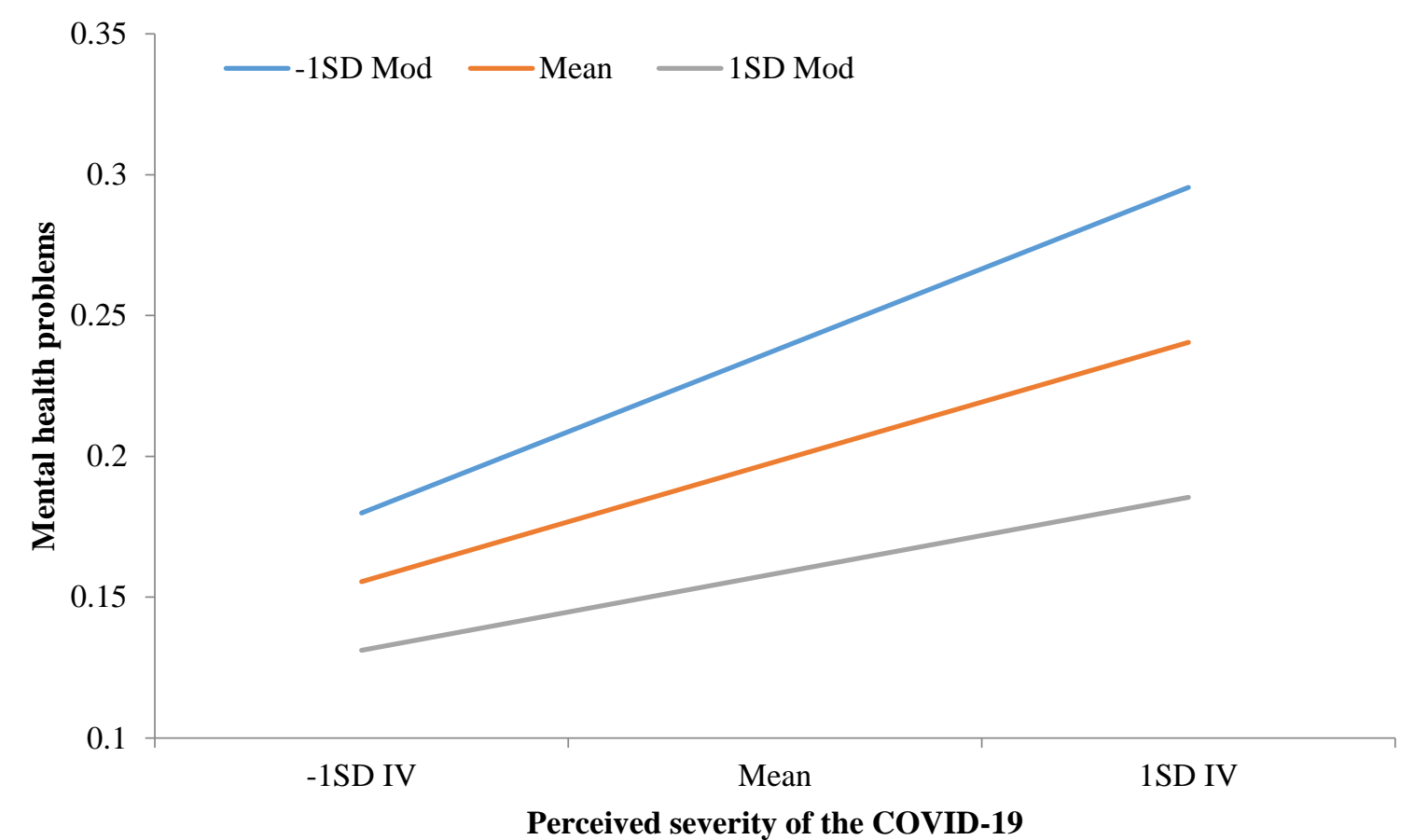

Figure 1 the association between perceived severity of the COVID-19 and meatal health problems by self-control. Note. IV = perceived severity of the COVID-19; Mod = self-control 